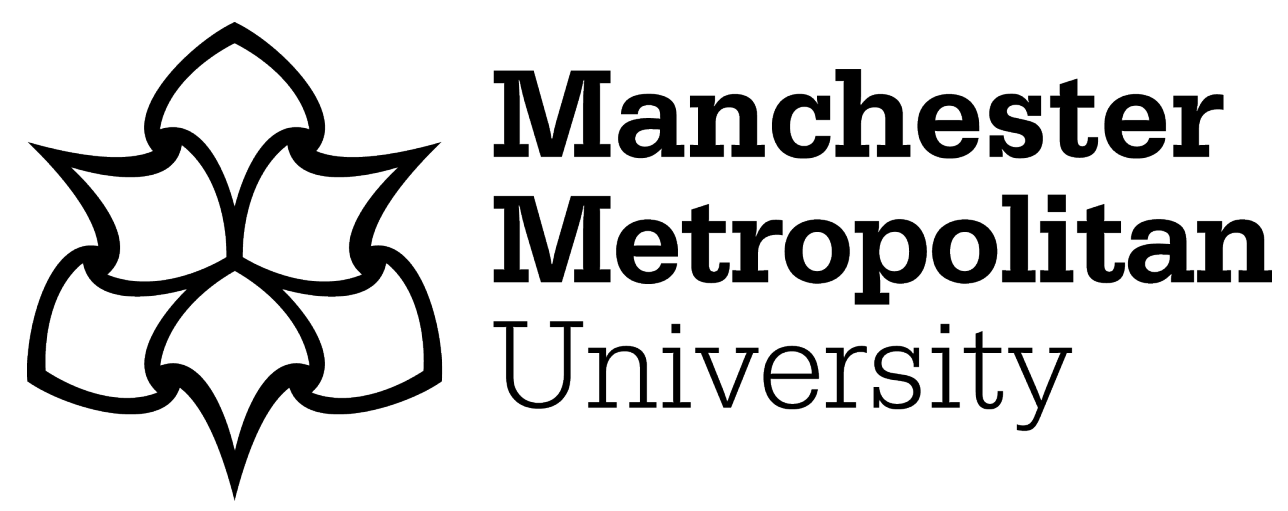

Lin, Zaibin, Qian, Ling ORCID logoORCID: https://orcid.org/0000-00029716-2342 and Bai, Wei ORCID logoORCID: https://orcid.org/0000-00023537-207X (2021) Numerical simulation of liquid sloshing using a fully nonlinear potential flow model in the non-inertial coordinate system. In: The 31st International Ocean and Polar Engineering Conference, 20 June 2021 - 25 June 2021, Rhodes, Greece.

Downloaded from: https://e-space.mmu.ac.uk/628074/

Version: Accepted Version

Please cite the published version 


\title{
Numerical simulation of liquid sloshing using a fully nonlinear potential flow model in the non-inertial coordinate system
}

\author{
Zaibin Lin, Ling Qian and Wei Bai \\ Centre for Mathematical Modelling and Flow Analysis, Department of Computing and Mathematics, \\ Manchester Metropolitan University, Manchester, M1 5GD, United Kingdom
}

\begin{abstract}
Liquid sloshing has been one of the primary concerns in ocean and offshore engineering due to its significant effects on ship stability and structure integrity. To investigate sloshing flow problems, a 3-dimensional Finite Volume Method based Fully Nonlinear Potential Flow (FNPF) model in the non-inertial coordinate system is developed in the present study. In this model, the Laplace equation is spatially discretised and solved using a second-order accurate finite volume method from the open source computational fluid dynamics software OpenFOAM. For the fully nonlinear free surface problems, both kinematic and dynamic boundary conditions at the free surface are implemented in the mixed-EulerianLagrangian (MEL) form to update the free surface elevation and velocity potential respectively. The FNPF sloshing model is validated against a number of available experimental measurements and numerical results for test cases under different external excitations. Finally, the conclusions in terms of model accuracy and applicability are summarised based on the validation and application results. It is found that the proposed FVM based sloshing FNPF model is able to simulate fully nonlinear liquid sloshing process in the non-inertial coordinate system.
\end{abstract}

KEY WORDS: OpenFOAM; Finite volume method; Liquid sloshing; Fully nonlinear potential flow; non-inertial coordinate system.

\section{INTRODUCTION}

Liquid sloshing has been a long-standing engineering issue for aerospace, civil, and marine engineering, and plays an important role in ensuring structural stability and safety. Violent structural motions induced by external conditions, such as launch and recovery of spacecrafts, ship motion especially LNG tankers under waves and wind, and skyscrapers under the combined effects of earthquake, wind, and other environmental sources, may lead to significant sloshing flows and impulsive loading on structure walls. These loads, in turn, may affect the stability and safety of structures and eventually cause structural damage to container walls, such as tank rupture. On the other hand, liquid sloshing in tanks has long been utilised as a vibration and motion control device and examples include the tuned liquid damper in civil engineering applications and anti-roll tanks for mitigating the roll motion of ships. Therefore, a robust, accurate, and effective numerical model, which is able to capture fully nonlinear free surface problems, is desired to estimate the fluid sloshing process inside a container.

Aiming at accurately capturing the complex fully nonlinear liquid sloshing process in a container, numerous numerical models based on Fully Nonlinear Potential Flow (FNPF) theory have been proposed and implemented using different numerical methods, such as Finite Difference Method (FDM) (Frandsen, 2004), Finite Element Method (FEM) (Wu et al., 1998; Kim et al., 2003; Wang and Khoo, 2005), Finite Volume Method (FVM) (Lin et al., 2019; 2021), Boundary Element Method (BEM) (Faitinsen, 1978; Nakayama and Washizu, 1981; Zhang, 2015), and Harmonic Polynomial Cell (HPC) (Shao, 2010; Liang et al., 2020). In these models, the free surface is updated based on the kinematic and dynamic boundary conditions and due to the potential flow assumption and its inherent limitations, they are not capable of capturing the violent post-wave breaking process such as free surface turbulence and air entrainment. However, compared to the two-phase viscous Navier-Stokes (NS) models based on Volume of Fluid (VoF) (Celebi and Akyildiz, 2002; Lee et al., 2007; Liu and Lin, 2008) or Level Set Method (LSM) (Gu et al., 2005; Chen and Price, 2009; Wang et al., 2011; Bai et al., 2015), the FNPF-based liquid sloshing model is far more computationally efficient (Ransley et al., 2019) under non-breaking sloshing scenarios.

Among these aforementioned FNPF-based sloshing models, Wu et al. (1998) adopted the FEM-based sloshing model to investigate the sloshing waves in a 3D tank and found that the occurrence of high pressure under certain circumstances may lead to significant damage to engineering structures. Subsequently, Wang and Khoo (2005) applied the FEM-based sloshing model to investigate the nonlinear sloshing problem due to random excitations, in which it was concluded that energy concentration may occur at the natural frequency of the container. Zhang et al. (2015) developed a BEM-based FNPF sloshing model to examine the second-order sloshing resonance in a 3-D container, which was further developed to simulate the sloshing process in an inverted trapezoid tank (Zhang, 2015). By extending the HPC-based FNPF model (Shao, 2010), Liang et al. (2020) incorporated an overset mesh 
technique to study liquid sloshing process under focused-wave-type excitations.

In this study, we propose a FVM-based liquid sloshing model in open access Computational Fluid Dynamics (CFD) software OpenFOAM by extending our in-house FNPF model for water wave problems (Lin et al., 2019; 2021). To better simulate the sloshing flow excited by potentially six degree-of-freedom external motions, such as a tuned liquid multi-column damper in Fath et al. (2020), the numerical model is constructed in the non-inertial coordinate system, which has the advantage of simplicity in dealing with body motions as well as implementing solid wall boundary conditions. In the following sections, the theory and governing equations for the FVM-based FNPF model are first described. Then a section dedicating to model validations against experimental measurements and other numerical results is presented to demonstrate the accuracy and robustness of the proposed model. Finally, the conclusions from this work are briefly summarised.

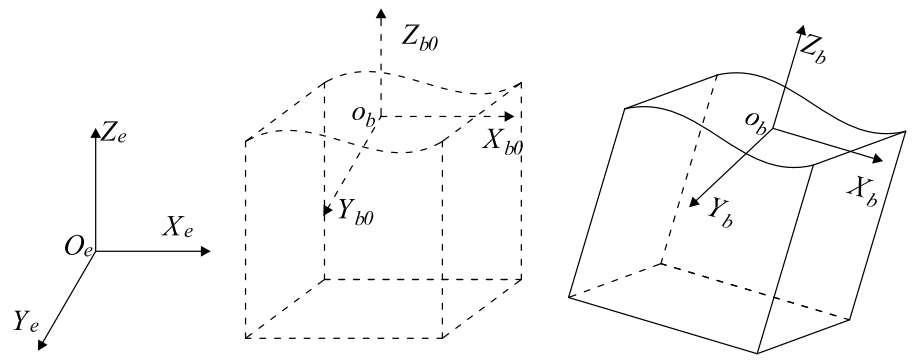

Fig. 1 Sketch of FNPF sloshing model.

\section{NUMERICAL MODELS}

In this section, based on the work in Shao (2010) the governing equation of fully nonlinear potential flow model in the earth-fixed coordinate system is introduced as follow:

$\nabla^{2} \phi=0$.

where $\phi$ is the velocity potential. In order to solve the Laplace equation for free surface problems in an earth-fixed coordinate system, the Kinematic Free Surface Boundary Condition (KFSBC) and Dynamic Free Surface Boundary Condition (DFSBC) must be satisfied:

$\frac{\partial \eta}{\partial t}=\frac{\partial \phi}{\partial z}-\frac{\partial \phi}{\partial x} \frac{\partial \eta}{\partial x}-\frac{\partial \phi}{\partial y} \frac{\partial \eta}{\partial y}$

$\frac{\partial \phi}{\partial t}=-\frac{1}{2} \nabla \phi \cdot \nabla \phi-\boldsymbol{g} \eta$

where $t$ is time, $\eta$ is wave elevation, $g$ is gravitational acceleration, ( $x$, $y, z)$ is in the earth-fixed coordinate $O_{e} X_{e} Y_{e} Z_{e}$ with $z$ pointing upwards. By prescribing that a point at free surface, e.g. the mesh cell vertex, can only move vertically, both KSFBC and DSFBC can be described in a semi-Eulerian-Lagrangian form:

$\frac{\delta \eta}{\delta t}=\frac{\partial \phi}{\partial z}-\frac{\partial \phi}{\partial x} \frac{\partial \eta}{\partial x}-\frac{\partial \phi}{\partial y} \frac{\partial \eta}{\partial y}$ $\frac{\delta \phi}{\delta t}=-\frac{1}{2} \nabla \phi \cdot \nabla \phi-\boldsymbol{g} \eta+\frac{\partial \eta}{\partial t} \frac{\partial \phi}{\partial z}$.

where $\frac{\delta()}{\delta t}$ is defined in the Lagrangian form as:

$\frac{\delta()}{\delta t}=\frac{\partial()}{\partial t}+\boldsymbol{U}_{m} \cdot \nabla()$

where $\boldsymbol{U}_{\boldsymbol{m}}=\left(0,0, \frac{\partial \eta}{\partial t}\right)$ is the prescribed velocity for a surface point and in the current work, it only has non-zero component in the $z$ direction. In the earth-fixed coordinate system, the pressure inside the computational domain is calculated through the Bernoulli equation:

$p=-\rho\left(\frac{\partial \phi}{\partial t}+\frac{1}{2} \nabla \phi \cdot \nabla \phi+\boldsymbol{g} z\right)$.

To investigate the fully nonlinear sloshing process induced by potentially multiple degree of freedom external excitations, a body-fixed coordinate system located in the free surface is adopted in the present study, as shown in Fig. 1. Thus, due to the transformation from the earthfixed coordinate system $O_{e} X_{e} Y_{e} Z_{e}$ to the body-fixed coordinate system $O_{b 0} X_{b 0} Y_{b 0} Z_{b 0}$ (initial location) and $O_{b} X_{b} Y_{b} Z_{b}$, both KFSBC and DFSBC are modified as follows:

$\begin{aligned} \frac{\delta \eta}{\delta t} & =\frac{\partial \phi}{\partial z}-\frac{\partial \phi}{\partial x} \frac{\partial \eta}{\partial x}-\frac{\partial \phi}{\partial y} \frac{\partial \eta}{\partial y}-\left[\boldsymbol{U}+\boldsymbol{\xi}^{\prime}+\boldsymbol{\omega} \times\left(\boldsymbol{r}-\boldsymbol{r}_{\boldsymbol{c}}\right)\right] \cdot\left(-\eta_{x},-\eta_{y}, 1\right) \\ & =\frac{\left\{\nabla \phi-\left[\boldsymbol{U}+\boldsymbol{\xi}^{\prime}+\omega \times\left(\boldsymbol{r}-\boldsymbol{r}_{\boldsymbol{c}}\right)\right]\right\} \cdot \boldsymbol{n}}{\boldsymbol{n}_{z}}\end{aligned}$

where $\frac{\boldsymbol{n}}{\boldsymbol{n}_{z}}$ equals to $\left(-\eta_{x},-\eta_{y}, 1\right)$.

$\frac{\delta \phi}{\delta t}=-\frac{1}{2} \nabla \phi \cdot \nabla \phi+\frac{\partial \eta}{\partial t} \frac{\partial \phi}{\partial z}+\left[\boldsymbol{U}+\boldsymbol{\xi}^{\prime}+\omega \times\left(\boldsymbol{r}-\boldsymbol{r}_{c}\right)\right] \cdot \nabla \phi-\boldsymbol{g} \cdot\left(\boldsymbol{\zeta}+R_{b \rightarrow c} \boldsymbol{r}\right)$.

where $\boldsymbol{U}$ is the forward speed of the body; $\boldsymbol{\xi}^{\prime}$ and $\boldsymbol{\omega}$ are the oscillatorily translational and angular velocities of the body described in the body fixed coordinate system $O_{b} X_{b} Y_{b} Z_{b}$ in Fig. $1 ; \boldsymbol{r}=(x, y, \eta)$ is the point at free surface relative to the origin of $O_{b} X_{b} Y_{b} Z_{b} ; \boldsymbol{r}_{c}=\left(x_{c}, y_{c}, z_{c}\right)$ is the position vector of the rotation centre $\left(x_{c}, y_{c}, z_{c}\right)$ relative to the origin of $O_{b} X_{b} Y_{b} Z_{b} ; \zeta=\boldsymbol{r}_{c}-R_{b \rightarrow c} \boldsymbol{r}_{c}$ and $R_{b \rightarrow e}$ is the transformation matrix in the order of yaw-pitch-roll Euler angles as follows:

$R_{b \rightarrow e}=A B C=\left[\begin{array}{ccc}c_{6} c_{5} & c_{6} s_{5} s_{4}-s_{6} c_{4} & c_{6} s_{5} c_{4}+s_{6} s_{4} \\ s_{6} c_{5} & s_{6} s_{5} s_{4}+c_{6} c_{4} & s_{6} s_{5} c_{4}-c_{6} s_{4} \\ -s_{5} & c_{5} s_{4} & c_{5} c_{4}\end{array}\right]$,

$A=\left[\begin{array}{ccc}c_{6} & -s_{6} & 0 \\ s_{6} & c_{6} & 0 \\ 0 & 0 & 1\end{array}\right], B=\left[\begin{array}{ccc}c_{5} & 0 & s_{5} \\ 0 & 1 & 0 \\ -s_{5} & 0 & c_{5}\end{array}\right] C=\left[\begin{array}{ccc}1 & 0 & 0 \\ 0 & c_{4} & -s_{4} \\ 0 & s_{4} & c_{4}\end{array}\right]$.

where $s_{i}=\sin \left(\alpha_{i}\right)$ and $c_{i}=\cos \left(\alpha_{i}\right)$ with $i=4,5,6$. In accordance with the transformation of coordinate system from $O_{e} X_{e} Y_{e} Z_{e}$ to $O_{b} X_{b} Y_{b} Z_{b}$, the pressure calculation based on the Bernoulli's equation also needs to be modified for the body-fixed coordinate system:

$\begin{aligned} p= & -\rho\left\{\frac{\partial \phi}{\partial t}+\frac{1}{2} \nabla \phi \cdot \nabla \phi+\left[\boldsymbol{U}+\boldsymbol{\xi}^{\prime}+\boldsymbol{\omega} \times\left(\boldsymbol{r}-\boldsymbol{r}_{\boldsymbol{c}}\right)\right] \cdot \nabla \phi\right. \\ & \left.+\boldsymbol{g} \cdot\left(\zeta+R_{b \rightarrow c} \boldsymbol{r}\right)\right\} .\end{aligned}$

where $p$ is pressure, $\rho$ is water density. 
For the solid wall boundary condition, it is given in the following form:

$\frac{\partial \phi}{\partial n}=\boldsymbol{n} \cdot\left[\boldsymbol{U}+\boldsymbol{\xi}^{\prime}+\boldsymbol{\omega} \times\left(\boldsymbol{r}-\boldsymbol{r}_{\boldsymbol{c}}\right)\right]$

As the kinematic and dynamic boundary conditions are described in the semi-Eulerian-Lagrangian form, the grids at the free surface are moved vertically based on Eqn. 8 and accordingly positions of internal nodes are also adjusted in the vertical direction. The first-order Euler explicit time scheme is used to discretise the unsteady term in the free surface boundary conditions, to update the wave elevation and velocity potential, and to estimate pressure in Eqn. 12. For more details on the implementation of the FNPF model, readers are referred to Lin et al. (2021).

\section{VALIDATIONS}

In this section, several test cases are selected to validate the present FNPF based sloshing model, including one 2-Dimensional (2-D) case under forced surge motion (Koh et al., 2012; Bai et al., 2015), two 2-D cases under forced pitch motion (Nakayama and Wushazu, 1980; Chen et al., 2013), and one 3-D case sloshing in a cylinder under forced pitch motion (Akyıldız et al., 2013).

\section{Forced 2-D surge excitation}

The developed model is firstly validated against the experiments performed in Koh et al. (2012) under horizontal excitations. The length and height of the water tank in laboratory are $0.6 \mathrm{~m}$ with water depth of $0.3 \mathrm{~m}$. A wave probe is located at $20 \mathrm{~mm}$ away from the right wall to measure free surface elevation and a pressure sensor is installed on the left wall at $0.02 \mathrm{~m}$ above the tank bottom. The horizontal displacement is theoretically controlled by

$x=A\left(1-\cos \left(\omega_{0} t\right)\right)$,

where $A$ is the surge amplitude, which is equal to $5 \mathrm{~mm}$, and $\omega_{0}$ is the natural frequency of the sloshing wave within the water tank and can be estimated by the following formula:

$\omega_{0}=\sqrt{g \frac{\pi}{L} \tanh \left(\frac{\pi}{L} d\right)}$,

where $L$ is the tank length and $d$ is the still water depth. A mesh sensitivity study is conducted first to assess the mesh convergence. Three different mesh setups are selected, including $25 \times 50,50 \times 50$, and $75 \times 50$, in the $x$ and $z$ directions, respectively, the mesh near the free surface is refined in the vertical direction as indicated in Fig. 2. The time step of the present model is determined by the Courant-Friedrichs-Lewy (CFL) condition, which is set to 0.3 in the current simulations. The results of these three mesh setups are compared with the experimental measurements under forced surge excitations (Koh et al., 2012) in terms of free surface elevation and pressure. In Fig. 3, it can be seen that the results from the FNPF model agree fairly well with the experimental data with the three different mesh setups, while only minor difference between the different mesh setups at $t=6.5 \mathrm{~s}-7 \mathrm{~s}$ is observed. A possible explanation for the discrepancy might be that the sloshing induced flow turbulence near the free surface are not captured by FNPF based models. The results of free surface elevation and pressure from the two finer meshes are almost identical indicating that a mesh converged solution can be achieved for the flow problem using $50 \times 50$ mesh cells.

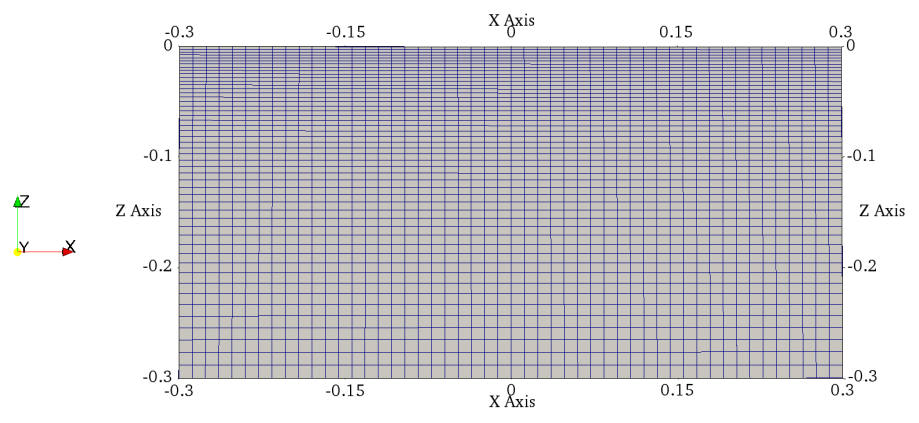

Fig. 2 Mesh setup for the case with 50x50 in the $x$ and $z$ directions, respectively.

(a)
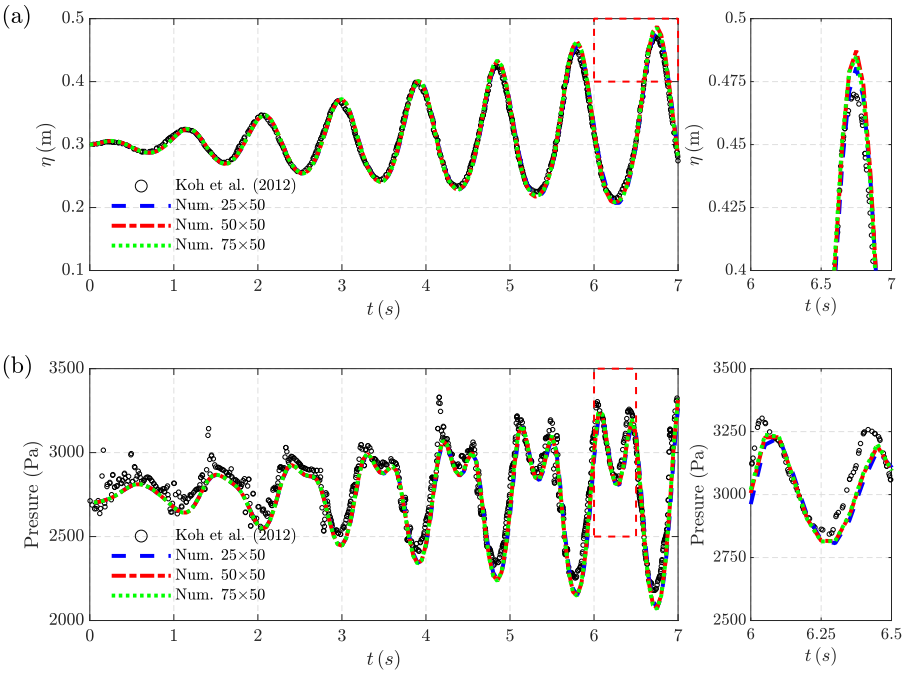

Fig. 3 Comparison of free surface elevation and pressure due to forced horizontal excitation: (a) Free surface elevation; (b) Pressure.

\section{Forced 2-D and 3-D pitch excitations}

In order to evaluate the accuracy of present model in capturing the water sloshing due to rotational excitations, two 2-D sloshing cases (Nakayama and Washizu, 1980; Zhao et al., 2018) in a rectangular water tank and one 3-D sloshing case in a cylindrical water tank (Akyıldız et al., 2013) are performed. The results are compared with other existing numerical results from the FNPF based on BEM (Zhao et al., 2018), the Smoothed Particle Hydrodynamics (SPH) (Chen et al., 2013), and the Navier-Stokes solver with Volume of Fluid (NS-VoF) in OpenFOAM (Xue et al., 2019), respectively.

In the first test case, the container has a length $(L)$ of $0.9 \mathrm{~m}$ and the still water depth $(D)$ is $0.6 \mathrm{~m}$. This container is subject to the following forced rotational oscillation:

$\theta(t)=\theta_{0} \cos \left(\omega_{r} t\right)$

where $\theta_{0}=0.8^{\circ}$ is the rotation amplitude; $\omega_{r}=5.5 \mathrm{rad} / \mathrm{s}$ is the rotational frequency. The rotation centre is located at the centre of the still water surface in the container and a mesh setup with $50 \times 1 \times 30$ cells in the $x, y$, and $z$ directions, respectively, has been used with mesh refinement near free surface as shown in Fig. 2. In Fig. 4, the free surface elevation at the left wall predicted by the current FNPF sloshing model are compared 
with the numerical results in Nakayama and Washizu (1980) and Zhao et al. (2018), showing good agreements, although slight difference at wave crest is presented.

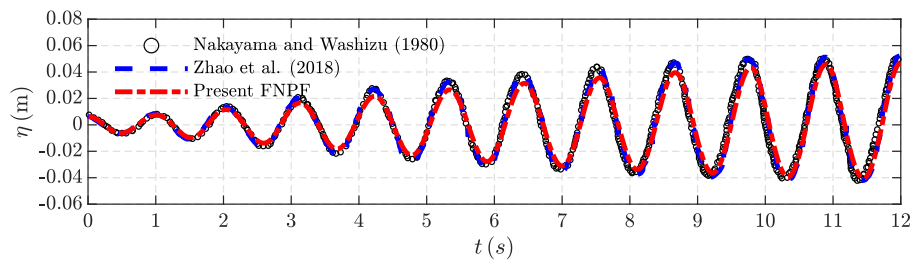

Fig. 4 Comparison of free surface elevation due to forced pitch excitation.

To further demonstrate the accuracy of the current FNPF model in terms of pressure field, experimental and numerical studies performed in Chen et al., (2013) are reproduced numerically. The fluid container has a length of $1 \mathrm{~m}$ and a width of $1 \mathrm{~m}$, and the still water depth is $0.3 \mathrm{~m}$, which is discretised using a mesh of $50 \times 50 \times 50$ cells with the mesh being well refined near the free surface. A sinusoidal rotational excitation is imposed at the centre of the container bottom wall:

$\theta(t)=\theta_{0} \sin \left(\omega_{r} t\right)$

where $\theta_{0}=5^{\circ}$ is the rotation amplitude and $\omega_{r}$ the rotation frequency. Two different rotation frequencies have been tested, one is $0.95 \mathrm{rad} / \mathrm{s}$ and the other is $3.09 \mathrm{rad} / \mathrm{s}$. In Fig. 5, the time series of pressure at $0.1 \mathrm{~m}$ below the initial free surface on the left wall are recorded and compared with the experimental measurements and the numerical results of SPH model in Chen et al., (2013), together with the BEM-based FNPF model results in Zhao et al. (2018). Compared to experimental measurements, the pressure at tough between $t=3-4 \mathrm{~s}$ in Fig. 5 is slightly underestimated. This may be attributed to the sloshing induced turbulence that cannot be predicted by both FNPF based sloshing models. In general, it can be seen that good agreements have been achieved between the current numerical results and other numerical and experimental results especially the one from the BEM-based FNPF model.

In addition, the liquid sloshing in a cylindrical container is simulated to examine the capacity of the present FVM-based FNPF model in modelling liquid sloshing in a non-rectangular container. To achieve this, the experiments in Akyıldız et al. (2013) are selected and numerically reproduced by the FVM-based FNPF model. The results are then compared with the experimental measurements and the numerical results from the Navier-Stokes solver in OpenFOAM (Xue et al., 2019). The diameter and height of the cylindrical container are $0.695 \mathrm{~m}$ and $0.8 \mathrm{~m}$, respectively. A sinusoidal rotational excitation (pitch) in Eq. 17 is imposed at the cylinder centre. Two rotation amplitudes of $\theta_{0}=4^{\circ}$ and $8^{\circ}$ with the same rotation frequency of $\omega_{r}=1.43 \mathrm{rad} / \mathrm{s}$ and water filling level of $25 \%$ have been tested.

In Fig. 6(a), it is clearly shown that the predicted pressure (at the point $0.05 \mathrm{~m}$ below the still water level with a $45^{\circ}$ azimuth angle from the negative $\mathrm{x}$ axis on the tank wall) from the FVM-based FNPF model agree well with the experimental measurements and numerical results from the NS-VoF model in OpenFOAM. It is worth noting that for the non-breaking sloshing test case, while the FVM-based FNPF model is computationally more efficient (Ransley et al.,2019; Lin et al.,2021), its results are almost identical to the ones from the NS-VoF model. For the larger rotational angle of $8^{\circ}$, the comparison between the numerical results using the present model and experimental measurements is
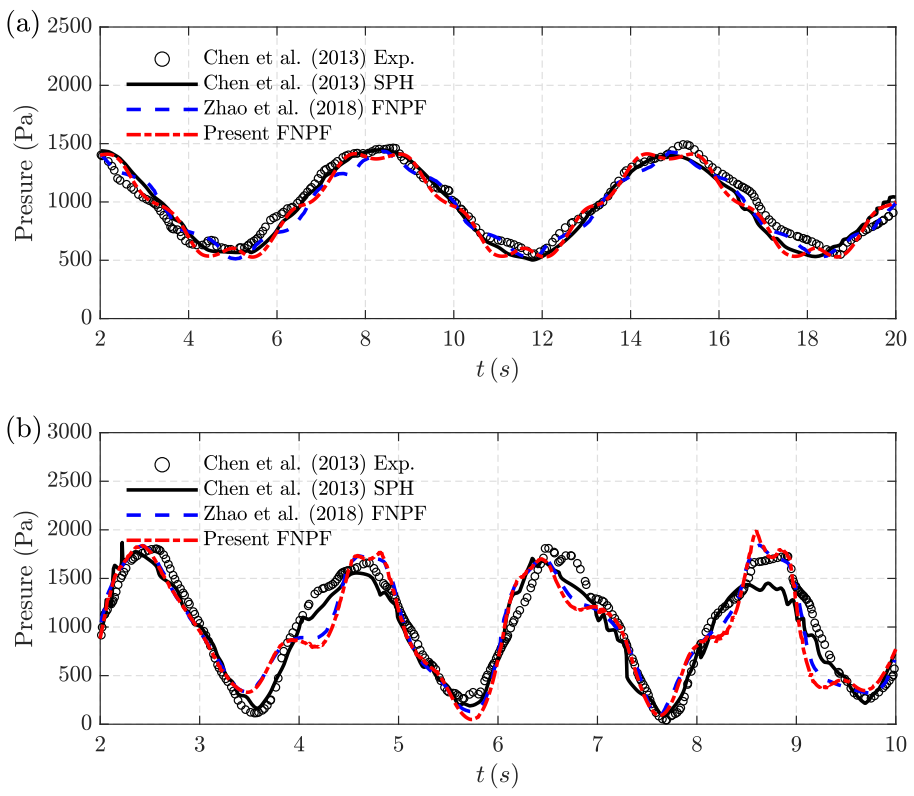

Fig. 5 Comparison of pressure due to forced pitch excitation with two different rotational frequencies: (a) $\omega=0.95 \mathrm{rad} / \mathrm{s}$; (b) $\omega=3.09 \mathrm{rad} / \mathrm{s}$.
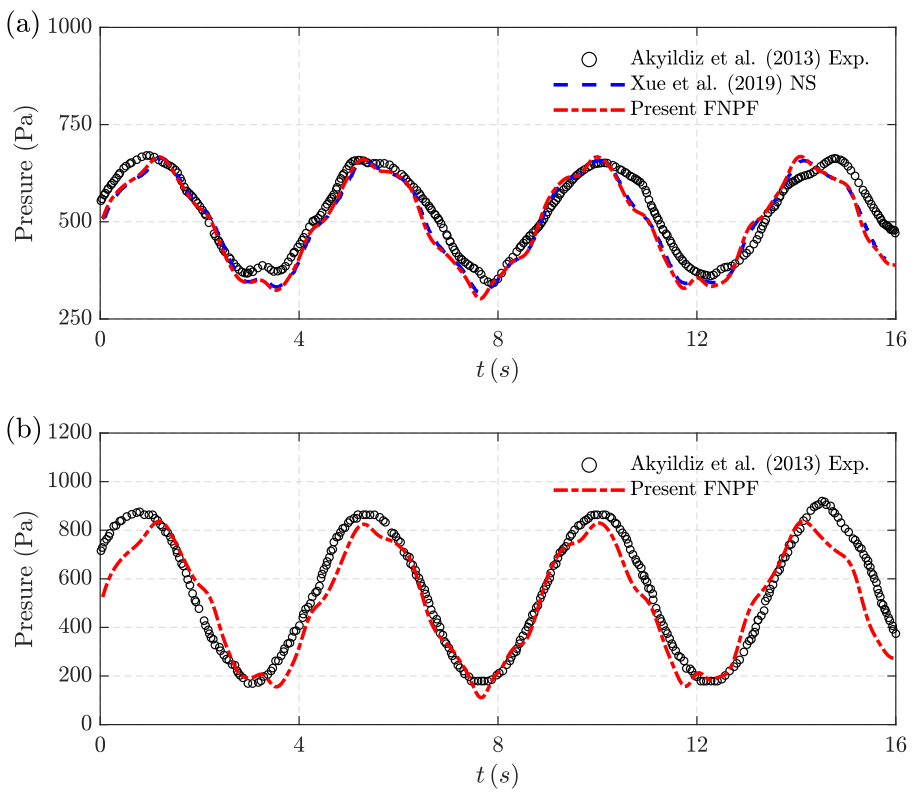

Fig. 6 Comparison of pressure in a cylindrical water tank due to forced pitch excitation with two different rotational excitation amplitudes: (a) $\theta_{0}=4^{\circ}$; (b) $\theta_{0}=8^{\circ}$.

shown in Fig. 6(b). While overall good agreements are achieved, some discrepancies between the results especially at the beginning and the end of the simulation can also be found. Further analysis on the sources of the discrepancy is still needed. The free surface elevations of the sloshing process for the case of $\theta_{0}=8^{\circ}$ at different moments in time are shown in Fig. 7, demonstrating the capability of the proposed FVM-based FNPF model in a body-fixed coordinate system for modelling liquid sloshing problems. 

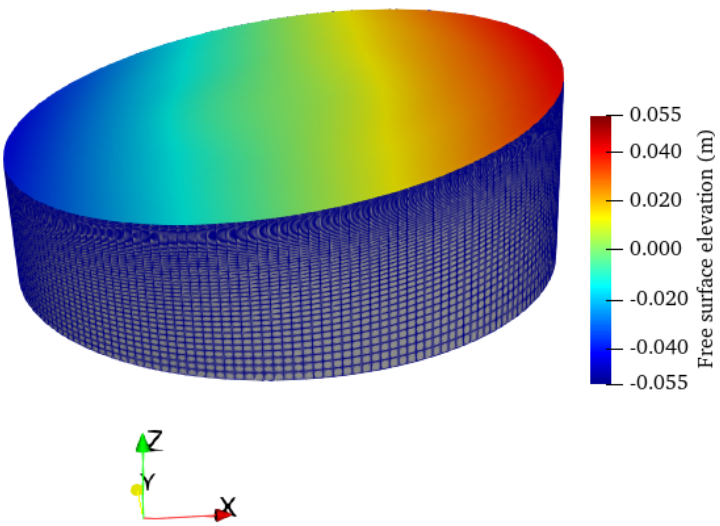

(a) Time: 3.05s
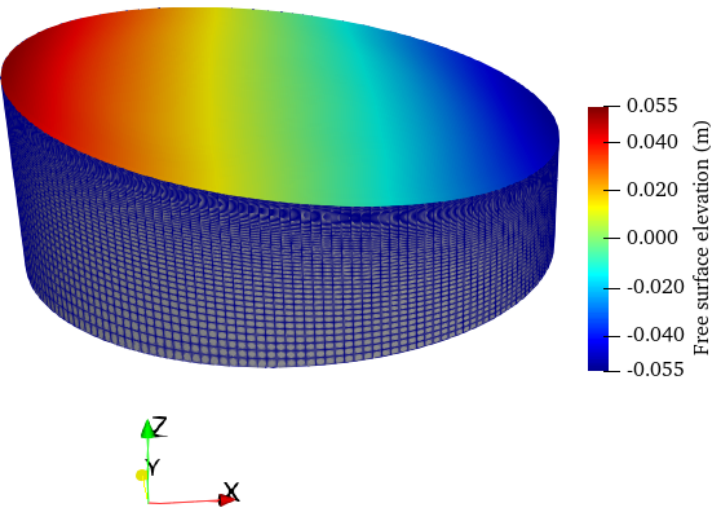

(b) Time: $5.35 \mathrm{~s}$
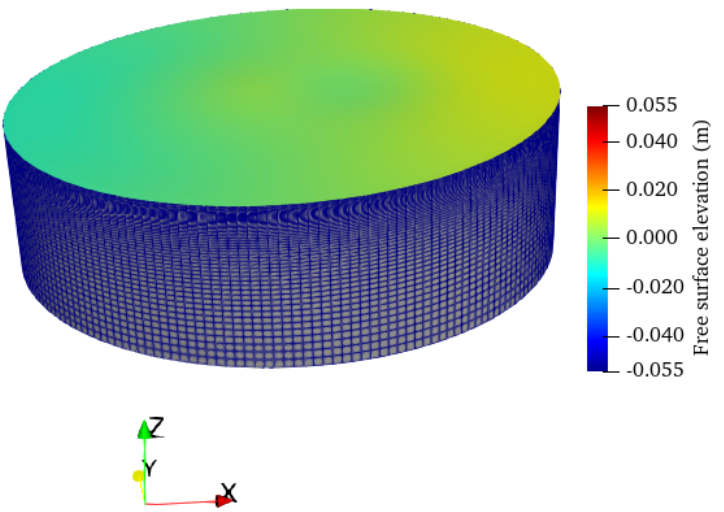

\section{(c) Time: $6.65 \mathrm{~s}$}

Fig. 7 Snapshots of free surface elevation of the sloshing in a cylindrical container for the case of $\theta_{0}=8^{\circ}$.

\section{CONCLUSIONS AND DISCUSSION}

In this study, a FVM-based FNPF model in the body-fixed coordinate system has been developed for liquid sloshing problems. The model is validated against existing experimental measurements and numerical results from various numerical methods, such as BEM-based FNPF model, SPH and NS-VoF models. In the test cases, forced translational and rotational excitations, i.e. forced surge and pitch motions, have been applied to the liquid containers, respectively. Excellent agreements have been achieved in terms of the time histories of the free surface elevation and pressure on the wall of both $2 \mathrm{D}$ rectangular and 3D cylindrical containers. This, along with the mesh refinement test and the achieved mesh converged solutions for the 2D sloshing in a rectangular tank, clearly demonstrates the accuracy and robustness of the proposed FVM-based FNPF model based on the body-fixed coordinate system for liquid sloshing problems. In the future, this newly developed model will be integrated into a NS-VoF solver so both wave interaction with floating offshore wind turbine support structures and the non-breaking liquid sloshing inside tuned liquid dampers can be modelled and their effectiveness on mitigating wave induced platform motions evaluated.

\section{ACKNOWLEDGMENTS}

This work was partially funded by the Engineering and Physical Sciences Research Council (EPSRC, UK) projects: Extreme Loading on FOWT under Complex Environmental Conditions (EP/T004150/1), A CCP on Wave Structure Interaction: CCP-WSI (EP/M022382/1) and the Supergen ORE Hub Flexible Fund project: Passive Control of Wave Induced Platform Motions for Semi-submersible FOWTs.

\section{REFERENCES}

Akyıldız, H, Ünal, NE, and Aksoy, H. (2013). An experimental investigation of the effects of the ring baffles on liquid sloshing in a rigid cylindrical tank. Ocean Engineering, 59, 190-197.

Bai, W, Liu, X, and Koh, C. (2015). Numerical study of violent lng sloshing induced by realistic ship motions using level set method. Ocean Engineering, 97, 100-113.

Celebi, MS, and Akyildiz, H. (2002). Nonlinear modeling of liquid sloshing in a moving rectangular tank. Ocean Engineering, 29(12), 1527-1553.

Chen, Y, and Price, W. (2009). Numerical simulation of liquid sloshing in a partially filled container with inclusion of compressibility effects. Physics of fluids, 21(11), 112105.

Chen, Z, Zong, Z, Li, H, and Li, J. (2013). An investigation into the pressure on solid walls in 2D sloshing using SPH method. Ocean Engineering, 59, 129-141.

Faitinsen, OM. (1978). A numerical nonlinear method of sloshing in tanks with two-dimensional flow. Journal of Ship Research, 22(03), 193-202.

Fath, A, Yazdi, EA, and Eghtesad, M. (2020). Semi-active vibration control of a semi-submersible offshore wind turbine using a tuned liquid multi-column damper. Journal of Ocean Engineering and Marine Energy, 6(3), 243-262.

Frandsen, JB. (2004). Sloshing motions in excited tanks. Journal of computational physics, 196(1), 53-87.

$\mathrm{Gu}, \mathrm{H}, \mathrm{Li}, \mathrm{Y}$, and Lin, P. (2005). Modeling 3D fluid sloshing using level set method. Modern Physics Letters B, 19(28n29), 1743-1746.

Kim, JKW, Shin, Y, Sim, I, Kim, Y, Bai, K, et al. (2003). Threedimensional finite-element computation for the sloshing impact pressure in lng tank. In The thirteenth international offshore and polar engineering conference.

Koh, C, Gao, M, and Luo, C. (2012). A new particle method for simulation of incompressible free surface flow problems. International Journal for Numerical Methods in Engineering, 89(12), 1582-1604.

Lee, D, Kim, M, Kwon, S, Kim, J, and Lee, Y. (2007). A parametric sensitivity study on lng tank sloshing loads by numerical simulations. Ocean Engineering, 34(1), 3-9. 
Liang, H, Santo, H, Shao, Y, Law, YZ, and Chan, ES. (2020). Liquid sloshing in an upright circular tank under periodic and transient excitations. Physical Review Fluids, 5(8), 084801.

Lin, Z, Qian, L, Bai, W, and Ma, Z. (2021). Simulation of steep focused wave impact on a fixed cylinder using fully nonlinear potential flow and navier-stokes solvers. International Journal of Offshore and Polar Engineering, 31(01), 78-86.

Lin, Z, Qian, L, Bai, W, Ma, Z, Chen, H, and Zhou, J-G. (2019). Development of 3-dimensional fully nonlinear potential flow planar wave tank in framework of OpenFOAM. In ASME 2019 38th International Conference on Ocean, Offshore and Arctic Engineering.

Lin, Z, Qian, L, Bai, W, Ma, Z, Chen, H, Zhou, J-G, and Gu, H. (2021). A finite volume based fully nonlinear potential flow model for water wave problems. Applied Ocean Research, 106, 102445.

Liu, D, and Lin, P. (2008). A numerical study of three-dimensional liquid sloshing in tanks. Journal of Computational physics, 227(8), 3921-3939.

Nakayama, T, and Washizu, K. (1980). Nonlinear analysis of liquid motion in a container subjected to forced pitching oscillation. International Journal for Numerical Methods in Engineering, 15(8), $1207-$ 1220.

Nakayama, T, and Washizu, K. (1981). The boundary element method applied to the analysis of two-dimensional nonlinear sloshing problems. International Journal for Numerical Methods in Engineering, 17(11), 1631-1646.

Ransley, E, Yan, S, Brown, S, Mai, D, T. Graham, Ma, Q, Musiedlak, P-H, Engsig-Karup, A, Eskilsson, C, Li, Q, Wang, J, Xie, Z, Sriram, V, Stoesser, T, Zhuang, Y, Li, Q, Wan, D, Chen, G, Chen, H, Qian, L, Ma, Z, Mingham, C, Causon, D, Gatin, I, Jasak, H, Vukčević, V, Downie, S, Higuera, P, Buldakov, E, Stagonas, D, Chen, Q, Zang, J, and Greaves, D. (2019). A blind comparative study of focused wave interactions with a fixed FPSO-like structure (CCP-WSI Blind Test Series 1). International Journal of Offshore and Polar Engineering, 29(2), 113-127.

Shao, Y-L. (2010). Numerical potential-flow studies on weaklynonlinear wave-body interactions with/without small forward speeds. Norwegian University of Science and Technology, Ph.D. Thesis.

Wang, C, and Khoo, B. (2005). Finite element analysis of two-dimensional nonlinear sloshing problems in random excitations. Ocean Engineering, 32(2), 107-133.

Wang, C-Y, Teng, J-T, and Huang, GP. (2011). Numerical simulation of sloshing motion inside a two dimensional rectangular tank by level set method. International Journal of Numerical Methods for Heat $\mathcal{F}$ Fluid Flow, 21(1), 5-31.

Wu, G, Ma, Q, and Eatock Taylor, R. (1998). Numerical simulation of sloshing waves in a $3 \mathrm{~d}$ tank based on a finite element method. Applied ocean research, 20(6), 337-355.

Xue, M-A, Chen, Y, Zheng, J, Qian, L, and Yuan, X. (2019). Fluid dynamics analysis of sloshing pressure distribution in storage vessels of different shapes. Ocean Engineering, 192, 106582.

Zhang, C. (2015). Application of an improved semi-lagrangian procedure to fully-nonlinear simulation of sloshing in non-wall-sided tanks. Applied Ocean Research, 51, 74-92.

Zhang, C, Li, Y, and Meng, Q. (2015). Fully nonlinear analysis of second-order sloshing resonance in a three-dimensional tank. Computers $\mathcal{E}$ Fluids, 116, 88-104.

Zhao, D, Hu, Z, Chen, G, Lim, S, and Wang, S. (2018). Nonlinear sloshing in rectangular tanks under forced excitation. International Journal of Naval Architecture and Ocean Engineering, 10(5), 545565 . 